\title{
Current Recommendations, Controversies, and Potential Novel Approaches in the Treatment of Wilms Tumor
}

\author{
Kevin Delijani ${ }^{1}$, Carolyn Hofley ${ }^{1}$, Nancy Luo ${ }^{1}$, George Yusin \\ ${ }^{1}$ Georgetown University School of Medicine \\ Keywords: pathophysiology, stem cell therapy, biomarkers, radiotherapy, partial nephrectomy, chemotherapy \\ https://doi.org/10.52504/001c.18059
}

\section{Georgetown Medical Review}

Vol. 5, Issue 1, 2021

\begin{abstract}
Wilms tumor (WT) is one of the most common renal malignancies in children, comprising about $5 \%$ of all childhood cancers. If diagnosed early, WT responds well to appropriate interventions such as surgical resection, chemotherapy and radiotherapy, with a 5 year survival higher than $85 \%$. In this review, we will describe the first-line treatment options for WT, discuss controversies related to particular modes of therapy, and highlight promising advances in molecular biology that may serve as effective therapeutics in the near future. Current treatment protocols for WT include nephrectomy followed by postoperative chemotherapy with or without preoperative chemotherapy. Though both are acceptable forms of treatment, preoperative chemotherapy prior to tumor resection has been shown to reduce tumor size and decrease the risk of intraoperative tumor rupture. Preoperative transcatheter arterial chemoembolization has also been shown to improve tumor resections and relapse-free survival rates, potentially providing an additive method to improve WT outcomes. Radical nephrectomy is the mainstay surgical treatment for WT, however evidence suggests that partial nephrectomies may be an equally viable option. Radiotherapy traditionally utilizes the anteroposterior-posteroanterior field technique, but recent advances have allowed for tumor-specific targeting and sparing of non-neoplastic tissues using intensity-modulated radiation therapy and volumetric-modulated arc therapy. Lastly, potential targets for future therapy include the $\beta$-catenin pathway, which has been found to be important in the development of WT, in addition to advances in applying microRNA, M6620, and stem cell therapy.
\end{abstract}

\section{Introduction}

Wilms tumor (WT), or nephroblastoma, is one of the most common renal malignancies encountered in the pediatric population. According to Szychot et al., WT comprises about $5 \%$ of all cancers typically diagnosed in childhood. ${ }^{1}$ The median age of WT manifestation is 3.5 years, and slight variations exist depending on ethnicity, gender, and genetic factors. ${ }^{1}$ Although many aspects of this disease remain unclear, strong correlates and risk factors have been identified that may predispose to and predict the development of WT. If diagnosed early, WT has been shown to respond well to appropriate interventions, in particular surgical resection, chemotherapy, and radiation therapy, with survival at 5 years being above $85 \% .{ }^{1,2}$ Although rare, cases of the adult form of WT typically have worse outcomes than traditional pediatric cases, primarily due to misdiagnosis as renal cell carcinoma, which in turn delays providing treatment specific for WT. ${ }^{1}$ The purpose of this review is to provide updates regarding mainstay treatment options, highlight controversies 
in the medical field regarding the order of therapeutic protocols, as well as discuss new therapeutic advances and discoveries that may help identify efficient treatment targets for WT in children and adults.

\section{Background}

\subsection{Pathology}

WT is considered to be derived from embryologic precursor lesions of the kidney called nephrogenic rests (NRs), which are noticed to have neoplastic changes. Two types of NRs are generally observed: intralobar (ILNRs) and perilobar (PLNRs). ILNRs can be located diffusely in the renal lobes, while PLNRs are typically found in the peripheral regions of the renal lobes. The predominance of each type of NRs has been shown to vary among different ethnic groups.

WT is known to be composed of three cell types: blastema, epithelium, and stroma. Identifying blastema cells is particularly important because a higher ratio of blastemal cells in WT classifies it as high risk. ${ }^{1}$ Several conventions exist on how to classify tumors based on histology, but WT is most often categorized into two groups, favorable histology and unfavorable histology/ anaplastic, based on the presence or absence of favorable outcomes produced by chemotherapy. Focal anaplasia is generally considered intermediate to high risk and easier to target than diffuse anaplasia. ${ }^{1}$

In addition to the two categories for WT histology described above, the International Society of Paediatric Oncology (SIOP) and Children's Oncology Group (COG) have developed a separate set of criteria that focuses more on the progression of WT, referred to as staging. Staging of WT, ranging from Stage I to Stage V, primarily depends on size, invasion into neighboring organs and vasculature, and unilateral or bilateral renal involvement. At Stage V, both kidneys have become involved and surgical resection is no longer an option. Furthermore, WT classification divides cases into two subclasses based on age and the likely histological manifestations. Type $1 \mathrm{WT}$ primarily affects young children, and has characteristics of ILNRs and stromal cells. ${ }^{1}$ Contrarily, type 2 WT typically manifests in older adults and contains PLNRs. ${ }^{1}$

\subsection{Genetics}

Genetics is thought to play a major role in determining the course of WT. According to Popov et al., anaplastic tumors are known to have an inactivated TP53 gene, which plays a role in the development of many other types of cancers aside from the kidneys. ${ }^{3}$ In addition, a number of genes are thought to strongly correlate with the development of WT. At this time, one of the strongest genetic associations in WT is a defect in the WT1 gene, which plays many important functions in encoding the respective transcription factor WT1 that directs the embryologic development of the kidneys and gonads. ${ }^{1}$ Deletion or disruption of WT1 is a strong predisposing factor for WT and is often associated with genitourinary abnormalities. ${ }^{1}$ Moreover, the WTX 
protein has been found to degrade $\beta$-catenin. ${ }^{4}$ A loss of WTX or an anomaly in the gene prevents degradation of $\beta$-catenin, leading to the constitutive activation of transcription factors further contributing to WT pathology. ${ }^{4}$ This will be discussed in more detail later in this paper. Interestingly, certain epigenetic changes, namely methylation of associated alleles, and patterns of genomic imprinting also determine WT gene expression. ${ }^{1}$

\subsection{Presentation and Evaluation}

WT typically presents in children less than 5 years old as an abdominal mass noted by the parents or by the pediatrician during a routine physical examination. While it is often asymptomatic, patients may present with abdominal pain, hematuria, and, in some cases, hypertension. ${ }^{1} \mathrm{WT}$ is initially diagnosed and observed with ultrasonography, then later confirmed via biopsy to assess for characteristic histological patterns. If not treated early on, WT can metastasize to other organs, which generally correlates with poorer prognosis. Since WT often metastasizes to the lungs, chest X-ray and/or CT scans are often recommended so that the appropriate treatment regimen can be given immediately to avoid further spread and associated complications. However, it is important to consider both the age of the patient and the risk factors for metastases in order to avoid unnecessary radiation delivered to a developing child. ${ }^{1}$

\section{Current Chemotherapy Treatments}

\subsection{Protocols and Treatment Recommendations}

The treatment protocol for newly diagnosed WT includes a combination of nephrectomy, chemotherapy, and radiotherapy based on a variety of factors including tumor histology, disease staging, and age of the patient. Currently, there are two accepted standards of treatment protocol for newly diagnosed WT as recommended by the COG and National WT Study Group (NWTSG), and SIOP. COG and NWTS protocols use the approach of initial nephrectomy followed by postoperative chemotherapy and radiation based on histologic grading and disease staging. ${ }^{5}$ SIOP, on the other hand, recommends preoperative neoadjuvant chemotherapy prior to nephrectomy (except in children less than 6 months of age) followed by postoperative chemotherapy and radiation. ${ }^{5}$ It is strongly recommended by all organizations that tumor histology is determined after surgical resection and that biopsy prior to nephrectomy is not performed except in cases of inoperable tumors and unusual clinical presentation. ${ }^{5}$ As stated in Clinicians' Practice Point guidelines in DynaMed, biopsy prior to nephrectomy or preoperative chemotherapy is not recommended for practicing physicians to perform in the case of Wilms tumor biopsy due to the risk of abdominal tumor cell spillage during biopsy that would result in additional radiation therapy and risk of abdominal metastasis. ${ }^{5}$ Since histologic grading and staging are performed after nephrectomy, staging guidelines followed by COG and SIOP differ in the 
fact that staging is performed prior to postoperative chemotherapy and after neoadjuvant chemotherapy, respectively, depending on the treatment protocol followed. ${ }^{5}$

\subsection{Chemotherapy Regimens}

Chemotherapeutic treatment regimens for WT include baseline medications of vincristine and dactinomycin for the majority of patients with additional agents added depending on the risk of recurrence and severity of disease stage. These additional chemotherapeutic agents include doxorubicin, epirubicin, cyclophosphamide, etoposide, carboplatin, ifosfamide and irinotecan. ${ }^{5}$ Combination therapies provided by COG and SIOP included in current treatment protocols have been established as best practice for different WT disease stages based on past and ongoing clinical trials to maximize tumor elimination and reduce the risk of recurrence. ${ }^{6}$ The two most current innovations in chemotherapy treatment for WT include the use of routine preoperative chemotherapy before surgical tumor resection in newly diagnosed WT cases and the potential implementation of preoperative renal transcatheter arterial chemoembolization (TACE) to improve complete tumor surgical resection and reduce relapse risk. ${ }^{7,8}$

\subsection{Neoadjuvant Chemotherapy}

Treatment protocol for newly diagnosed WT remains a controversial topic among international oncology groups, with the main difference between the two protocols being preoperative chemotherapy prior to tumor surgical resection and histologic staging, verses nephrectomy and histological staging prior to postoperative chemotherapeutic treatment, as recommended by the SIOP and COG respectively. The main argument in favor of preoperative chemotherapy is that it significantly decreases tumor size prior to surgical resection which reduces the risk of tumor rupture during surgery. As a result, the risk of metastases and tumor recurrence is significantly diminished. ${ }^{7,9}$ The SIOP argues that reduction in tumor size prior to surgery allows for a renal sparing surgical approach and decreases the need for postoperative chemotherapy and radiation. ${ }^{7}$ The COG recommends an initial nephrectomy approach without preoperative chemotherapy, except in cases of advanced disease (e.g. multicentric tumor, bilateral tumor, solitary kidney, or inoperable tumors) with the argument being that preoperative chemotherapy alters the initial tumor histology and therefore compromises staging information, potentially resulting in downstaging of the tumor. ${ }^{5,7}$ Despite this, it is recognized that nephrectomy without preoperative chemotherapy increases the risk of tumor rupture during surgery and subsequent relapse from tumor cell spillage into the abdominal cavity. 7 A separate pediatric oncology society, the United Kingdom Children's Cancer Study Group (UKCCSG) conducted a head-to-head clinical trial comparing the treatment protocols developed by the COG and SIOP.' The study found that the use of preoperative chemotherapy reduced both the likelihood of surgical complications from intraoperative 
tumor rupture and the amount of postoperative chemotherapy and radiation needed. ${ }^{10}$ Furthermore, the tumors were more likely to show favorable histological changes and decreased staging. 7,10 These findings support the preoperative chemotherapy treatment protocol for WT set forth by the SIOP. Moreover, the UKCCSG now joins the SIOP's recommendations in ongoing clinical trials for WT treatment. ${ }^{7,10}$ Interestingly, the COG recently conducted a clinical trial looking at the effect of combination preoperative chemotherapy on reducing preoperative tumor size and the amount of functional kidney tissue removed, but the results are still awaiting publication. ${ }^{6}$ Nonetheless, the preliminary results show favorable patient outcomes for patients who had preoperative chemotherapy prior to nephrectomy, a finding that may change the current treatment protocols for WT in the United States. ${ }^{6}$

\subsection{Transcatheter Arterial Chemoembolization}

Renal transcatheter arterial chemoembolization (TACE), a technique where blood supply to a tumor is blocked using chemotherapeutic agents, has recently been used as a newer ancillary treatment approach to improve WT resection outcomes. Currently, TACE is not part of the recommended WT treatment protocol put forth by the SIOP and COG. ${ }^{5}$ However, studies suggest that TACE should be added as part of the chemotherapeutic treatment protocol prior to WT resection because of its favorable outcomes in WT patients. ${ }^{8}$ One such study showed that preoperative TACE in WT patients resulted in higher rates of complete tumor removal and relapse-free survival compared to preoperative chemotherapy. ${ }^{8}$ Additionally, the study also found that the combination of TACE and preoperative systemic chemotherapy achieved even higher rates of resection and relapse-free survival, suggesting that the addition of TACE to the current SIOP neoadjuvant chemotherapy treatment protocol could prove beneficial in WT patients. ${ }^{8} \mathrm{~A}$ more recent study even found that TACE was more effective at achieving complete tumor resection by inducing higher rates of tumor necrosis, leading to a greater decrease in tumor size and improved overall survival rates compared to standard preoperative chemotherapy alone. ${ }^{11}$ From these studies, TACE should be further explored as a viable addition to the current WT treatment protocols, and may be a future component of treatment guidelines for WT.

\section{Current Partial Nephrectomy Treatment}

Currently, the United States follows the recommendations laid out by the COG guidelines, so upfront resection prior to chemotherapy remains the cornerstone treatment for WT. ${ }^{5}$ The most common surgery in WT is radical nephrectomy, where the whole kidney, surrounding fat, and adrenal glands are removed. Partial nephrectomy, or nephron-sparing surgery, is less common due to the concern for local recurrence and the likelihood that the tumor is too large once discovered. However, a systematic review of partial nephrectomy articles revealed that radical nephrectomy and partial nephrectomy in children with WT show similar recurrence rates $(12 \%$ vs. $11 \%)$ and survival rates $(85 \%$ 
vs. $88 \%) .{ }^{12}$ These results may be inaccurate due to inconsistent reporting and small sample sizes of partial nephrectomy cases at most institutions. Nonetheless, there have been reported benefits of partial nephrectomies over radical nephrectomies in WT, such as a reduced risk for developing severe chronic kidney disease. ${ }^{12}$ Given that current studies seem to support partial nephrectomies as a viable surgical option for WT, the next step would be to develop guidelines for patient groups that may benefit from such treatment.

\section{Current Adjunctive Radiotherapy Techniques for Favorable Histology Stage III-V or Diffuse Anaplastic W'T}

Radiotherapy significantly improves the overall survival of patients with WT lung metastases, as shown by the NWTS-3 and 4 which found that the overall survival rate was $91 \%$ after whole lung irradiation compared to $85 \%$ with just chemotherapy alone. ${ }^{13}$ According to NWTS guidelines, Stage III favorable histology and Stage II-III anaplastic WT should receive abdominal radiation of 10.8 Gray $(\mathrm{Gy}) .^{13}$ For patients with pulmonary metastases, whole-lung radiation of 12 Gy is used. ${ }^{13}$ Radiotherapy in WT has traditionally been accomplished using the anteroposterior-posteroanterior (AP-PA) photon field technique where patients receive a uniform dose of radiation to both neoplastic and surrounding normal tissue. ${ }^{14}$ However, with the advent of new imageguided radiation delivery systems within the past several years, WT can now be more precisely targeted in order to limit potential long-term sequelae from flank or abdominal radiotherapy.

\subsection{Intensity-Modulated Radiation Therapy}

Intensity-modulated radiation therapy (IMRT) is a technique where photon beam intensity is modulated based on the arrangement of the target tissue and surrounding organs. It has the ability to decrease the intensity of radiation that passes through normal tissue and increase the intensity of radiation that passes through neoplastic tissue. IMRT is unique in that it allows clinicians to control the 3-dimensional dose distribution from a fixed number of radiation fields, ultimately shaping radiotherapy to the geometric conformation of the tumor. ${ }^{15}$

One recent study evaluated the use of IMRT in cardiac-sparing whole lung irradiation across a multi-institutional setting. ${ }^{15} \mathrm{~A}$ total of 20 patients with lung metastases were recruited into the study, 5 of whom were patients diagnosed with WT. ${ }^{15}$ The results from this study showed a significantly lower mean radiation dose to the whole heart and coronary arteries using IMRT, but no change in mean dose to the thyroid gland, liver, stomach, spleen, and stomach compared standard AP-PA techniques. ${ }^{15}$ Two-year follow-up after radiotherapy revealed no reports of pulmonary toxicity or radiotherapy-related lung consolidations. ${ }^{15}$ This multicenter report was critical for confirming the cardio-protective advantage of IMRT over the standard AP-PA radiotherapy for lung metastases and is anticipated to be incorporated into upcoming WT treatment protocols. ${ }^{15}$ Despite the seemingly superior results of IMRT 
treatment, there is still concern for lower dose radiation spillage into normal tissue which can result in the late development of secondary malignancies. One drawback of this study is the lack of long-term follow-up on patients to determine the risk of developing late complications from IMRT, such as heart failure, pulmonary disease, and secondary malignant neoplasms. ${ }^{15}$

\subsection{Volumetric-Modulated Arc Radiotherapy}

Volumetric-modulated arc radiotherapy (VMAT) is considered an extension of IMRT in that, likewise, it delivers accurate radiation dose distributions to the tumor, but instead of having a fixed plane, the radiotherapy machine can rotate 360 o around the patient. One criticism of IMRT is that its daily treatment time is longer than that of non-modulated 3D planning because it requires a higher number of motor units to achieve the desired conformal dose distribution around the target tissue. This poses an issue for many young children with WT in that it increases the need for sedation to prevent movement and consequently, inaccurate radiation delivery. Even in patients who are not sedated, longer treatment sessions increase the chance for erroneous radiation dosage to healthy tissue if the patient moves. VMAT addresses this issue by providing more beam entry angles, thereby reducing the number of motor units required and decreasing treatment session times. A few case studies have been reported using VMAT to treat WT lung metastases in an effort to decrease radiation to organs traditionally affected by AP-PA field radiation. One such case involved a 3-year-old girl diagnosed with Stage I WT who was initially treated with complete resection of the primary tumor and adjuvant chemotherapy. ${ }^{14}$ One month after chemotherapy, CT scans revealed bilateral lung metastases which were treated with VMAT whole-lung irradiation using a standard total prescription dose of 12 Gy. ${ }^{14}$ VMAT displayed significant advantages over AP-PA field techniques by reducing the mean dosage to the heart, liver, and thyroid gland by 3.8 Gy, 3.7 Gy, and $6.6 \mathrm{~Gy}$ respectively. ${ }^{14}$ Furthermore, radiation delivered by VMAT was well-tolerated and did not induce any subsequent treatment-related toxicities. ${ }^{14}$ This case study highlights the utility of VMAT in reducing radiation toxicity to normal tissues and organs - a critical advancement that may decrease morbidity and mortality rates associated with radiotherapy.

\subsection{Intensity Modulated Proton Therapy}

Similar to IMRT, intensity modulated proton therapy (IMPT) is designed to modulate the amount of radiation given to the surrounding healthy tissue near a tumor using proton particles instead of photons. The advantage of using proton over photon therapy is that protons reduce damage to non-neoplastic cells and do not have an exit point, instead stopping once they reach an energydependent distance. ${ }^{16}$ To our knowledge, there have been no published reports or studies that used IMPT in the treatment of WT. However, one research group has proposed a pencil-beam scanning (PBS) proton therapy for WT patients following nephrectomy. ${ }^{17}$ Though untested, the idea behind PBS 
proton therapy is to provide greater control of radiation to concave structures in the retroperitoneum while minimizing dosage to surrounding healthy tissues compared to AP-PA flank or whole lung irradiation. This study used the planning CT images obtained from 11 patients with WT who received AP-PA radiation to construct PBS proton therapy plans. ${ }^{17}$ Compared to the AP-PA dosage plans, PBS proton therapy plans had significantly reduced mean doses to the contralateral kidney, bowel, and liver, but not the pancreas. ${ }^{17}$ These findings suggest clinical utility for future treatment of WT using PBS proton therapy, however more research will be needed to translate this hypothesis into reality.

\subsection{Comparing Radiotherapy Treatments in WT}

Regarding WT specifically, there is a paucity of studies comparing imageguided radiotherapy techniques. One benchmark study involving four WT patients demonstrated that IMPT treatment planning was better than that of photon beam IMRT at reducing radiation dosage to normal tissue in the low to medium dose range, defined as $<50 \%$ of the dose delivered to the tumor. ${ }^{16}$ We believe that one way to address the lack of comparative radiotherapy studies in WT is to create a core database that tracks treatment parameters along with patient characteristics so that guidelines comparing different image-guided planning techniques can be created.

Another important factor to consider when assessing the clinical value of radiotherapy treatments is the type of image modality used to outline the margins of a retroperitoneal tumor. MRI-guided radiotherapy has been recently introduced into clinics and is suggested to be superior to traditional CT-guided radiotherapy due to its higher soft-tissue resolution and radiationsparing imaging. ${ }^{18}$ By using real time MRI-guided IMRT as opposed to conebeam CT VMAT, the planning target volume margin of the tumor can be reduced up to $2 \mathrm{~mm}$ in pediatric patients with WT without affecting the final cumulative dose to the tumor. This margin reduction results in a significant decrease in mean radiation dose by $3.4 \%, 2.8 \%$, and $4.9 \%$ to the liver, spleen, and pancreas, respectively - organs which are commonly impacted by flank radiation and contribute to the development of metabolic syndrome, intestinal occlusion, and secondary cancer in patients who undergo radiotherapy. ${ }^{18}$

\section{Potential Therapies}

\subsection{Wnt/ $\beta$-catenin Pathway}

To date, the Wnt/ $\beta$-catenin pathway has been well characterized and associated with embryogenesis and neoplastic conditions as a proto-oncogene. As mentioned in the discussion of WT genetics, a loss or mutation in the WTX gene contributes to the development of WT and leads to the unregulated activation of $\beta$-catenin. Roughly $15-20 \%$ of all non-anaplastic WT contain signs of activation of the $\mathrm{Wnt} / \beta$-catenin pathway. ${ }^{4}$ 
Wnt regulates the phosphorylation and subsequent degradation of $\beta$-catenin. When Wnt is present, it binds to a Frizzled receptor and its co-receptor, causing the recruitment of Dishevelled which destabilizes the degradation complex by preventing its phosphorylation and allowing $\beta$-catenin to potentiate its effects. ${ }^{19,20}$ When Wnt is absent, $\beta$-catenin forms a stable complex with other mediators, such as Axin, APC, GSK3, and CK1, which facilitates the phosphorylation of $\beta$-catenin to signal for proteasomal degradation. ${ }^{21}$ Therefore, a deletion of APC or mutations in the serine and threonine amino acids of $\beta$-catenin inhibits the degradation complex from forming, allowing $\beta$-catenin to escape deactivation. 22 When Wnt is present and $\beta$-catenin accumulates within the cell, it is able to enter the nucleus via an undetermined mechanism to activate transcription of the TCF/ LEF family, which upregulates transcription factors such as Cyclin $\mathrm{B} 1$ and Cyclin $\mathrm{C}$, promoting carcinogenesis. ${ }^{23,24}$ The $\mathrm{Wnt} / \beta$-catenin pathway is also closely related with the embryological development of the kidney. ${ }^{25}$ According to Perotti et al., Wnt signaling is required to initiate nephrogenesis and mesenchymal-to-epithelial transition, which is necessary for metanephric mesenchyme to establish the epithelial tubules that form the mature nephron. Additionally, abnormal Wnt signaling is closely related to WT development. ${ }^{4}$

Another very common mutation associated with WT development is a constitutively activated Wnt/ $\beta$-catenin pathway caused by a CTNNB1-activating mutation. ${ }^{4}$ There are multiple options for therapeutic intervention by blocking the Wnt/ $\beta$-catenin pathway, such as inhibiting ligand production, blocking the Frizzled receptor, inhibiting $\beta$-catenin transport into the nucleus, or blocking $\beta$-catenin interaction with $\mathrm{c}$-terminal cofactors. ${ }^{26}$ Frizzled 7 is one such receptor target of Wnt, and WT has been characterized as sensitive to FZD7 (an antibody against Frizzled 7), or resistant. ${ }^{27}$ PodeShakked et al. grafted mammalian cancer cells to the chorioallantoic membrane of the fertile chick egg as a xenograft system to determine the effect of FZD7 antibody on the growth and proliferation of FZD7- sensitive WT cells in vivo. ${ }^{27}$ Grafted human WT cells were incubated with FZD7 antibody or buffer (as the control) and stained with Ki67 antibody, a mammalian specific antibody used to detect cancer cells. ${ }^{27}$ The results of this experiment indicated that FZD7 antibody, which blocks the Wnt/ $\beta$-catenin pathway, reduces the survival and proliferation of FZD7 sensitive WT in vivo. ${ }^{27}$ Currently, there are many potential therapies targeting the $\beta$-catenin pathway that are in early clinical or preclinical stages.

There are no clinical trials underway at this moment to determine the effectiveness of blocking the Wnt/ $\beta$-catenin pathway in treating WT, but this method is being explored to treat other malignancies. For instance, tegavivint is a $\beta$-catenin/transducing $\beta$-like protein 1 inhibitor that has antitumor activity in metastatic osteosarcoma in vivo. ${ }^{28}$ The prospective clinical benefits these therapies may have in treating WT are promising as they are further investigated. 


\subsection{MicroRNAs}

MicroRNAs (miRNAs) are single-stranded RNAs that can posttranscriptionally alter gene expression by promoting mRNA degradation or inhibiting translation. Carcinomas of the kidney have been shown to express certain miRNAs in a patient's serum or plasma, which may be analyzed for diagnostic or prognostic purposes. ${ }^{29,30}$ Ludwig et al. analyzed the overexpression of 19 miRNAs in the serum of patients with WT. ${ }^{29}$ A significantly higher expression of miRNAs-100-5p, -130b-3p, and -143-3p was identified before the onset of therapy. ${ }^{29}$ This set of miRNA signatures offered an accuracy of $84.58 \%$ for differentiating WT patients from healthy controls. ${ }^{29}$ Additionally, miRNAs offer additional clinical value in their ability to predict the prognosis of treatment. When the miRNA profile of 132 WT patients and 6 normal samples were analyzed, it revealed that $18 \mathrm{miRNAs}$ had an association with overall survival and 5 differentially expressed miRNAs were able to classify the patients into high or low risk groups. ${ }^{31}$ Not only does this non-invasive method of diagnosing and prognosticating WT have high clinical value for the future, but it also may delineate any differential expression of miRNAs that can be used as potential targets of therapy.

miRNAs play a significant role in regulating many biological processes such as cell cycle progression, cell survival, differentiation, and development. As a result, if the expression of miRNAs is modulated, it may affect the proliferation and course of human diseases such as genetic disorders, autoimmune disorders, and cancer. ${ }^{32} \mathrm{~A}$ common aberrancy prevalent in WT is mutations in miRNA processing genes, as roughly $15 \%$ of WT patients have mutations in these genes. ${ }^{30}$ Furthermore, miRNAs have been shown to be able to upregulate or downregulate the differentiation of WT, indicating their involvement in WT pathology. One example of this is miR-200c-3p, which was shown to be downregulated in nephroblastoma tissue and to inhibit the proliferative, migratory, and invasive abilities of WT cells by targeting fibroblast growth factor receptor substrate $2 .{ }^{33}$

Currently, the clinical applicability of miRNAs in cancer treatment is under ongoing investigation. Though there are no clinical trials yet involving miRNA targets for WT, there is a Phase 1 clinical trial underway to determine the efficacy of Selinexor, an XPO1 inhibitor, in the treatment of solid tumors. ${ }^{34}$ $\mathrm{XPO} 1$ is a protein which plays a role in the synthesis of mature miRNA. This clinical trial is currently in the process of collecting patients. ${ }^{34}$

\section{$6.3 M 6620$}

Among the new emerging methods for treating childhood cancers, including favorable histology WT, Rad3-related kinase (ATR) inhibitors have been shown to sensitize cancer cells to concomitant chemotherapy regimens. ${ }^{35}$ ATR, alongside a few other kinase proteins, plays a crucial role in stabilizing the cell genome and forms the DNA damage response mechanism. ${ }^{35,36}$ Thus, Thomas et al. suggest that targeting these enzymes can significantly increase 
the efficacy of chemotherapy drugs by inhibiting the DNA repair mechanisms in malignant cells. M6620, a recently developed agent, inhibits ATR with high selectivity and potency. ${ }^{36}$ Kurmasheva et al. tested M6620 in vitro and observed that although it had little efficacy when used alone, the combination of standard chemotherapy regimen with M6620 exerted a substantial cytotoxic effect, effectively inhibiting growth of various carcinomas, including WT. ${ }^{35}$ Thomas et al. further described a Phase I clinical trial testing the effect of the combined M6620/ topotecan (TOP1 inhibitor) therapy in patients with advanced solid tumors. ${ }^{36}$ Since this combination was successful in stabilizing and improving patient outcomes, such as sufficient antitumor activity, safety, and tolerability, it has moved on to Phase II of clinical trials. ${ }^{36}$

\subsection{Stem Cell Therapy}

Few studies are available that describe the efficacy of stem cell therapy for WT patients. Lee et al. have described the limited benefits of autologous stem cell transplantation (ASCT) used in conjunction with common chemotherapy regimens. ${ }^{2} \mathrm{WT}$ is typically effectively treated and targeted with chemotherapy in childhood, however, it is common to see cases of WT relapse in adulthood. For this reason, the benefits of stem cell rescue and bone marrow transplant to replace hematopoietic stem cells damaged during the course of chemotherapy have been explored. ${ }^{2}$ While monitoring renal and tubular function in WT participants, it was found that glomerular function remained intact following a regimen of high dose chemotherapy (HDCT) in the form of carboplatin along with ASCT (HDCT/ ASCT). ${ }^{2}$ This is particularly important due to the fact that many patients treated for WT with this regimen present with one kidney post-nephrectomy, thus reinforcing the importance of kidney preservation. ${ }^{2}$ Malogolowkin et al. suggest that the addition of ASCT is generally welltolerated and improves survival in young WT patients who received HDCT and experienced relapses later on in life. ${ }^{37}$ However, many of the adverse outcomes, such as tumor relapse, transplant-related mortality, and transplant failure, may still be present. ${ }^{37}$ It was also noted that many practitioners are currently shifting their focus from studying bone marrow stem cell transplant to peripheral blood stem cell transplant, which may be more effective in treating WT and other conditions. ${ }^{37}$ Thus, further research into stem cell therapy as an adjunctive treatment method for WT is warranted and may produce favorable patient outcomes while achieving full response to HDCT/ ASCT.

\section{Conclusion}

In summary, this review aimed to highlight currently available treatment modalities for WT, discussing their advantages and areas of improvement. This manuscript also describes promising cutting-edge research methods and findings that are making valuable contributions to the development of novel therapeutics. These advances have the potential to be tested further and used to treat pediatric nephroblastoma. Curative treatments for WT currently revolve 
around a combination of chemotherapy, nephrectomy, and radiotherapy depending on the stage of the tumor. Based on the risk of tumor cell spillage during surgery, we recommend following the SIOP guidelines of implementing chemotherapy before nephrectomy in order to decrease both the size and stage of the tumor prior to performing a resection. Additionally, the use of TACE prior to surgical resection may be a potential future avenue to further improve complete tumor resection and decrease relapse risk. Post-nephrectomy chemotherapy should also be utilized in order to destroy any remaining WT cells that were missed or spilled during surgery. If a patient presents with favorable histology low-stage WT, we recommend that clinicians consider performing a partial nephrectomy over a radical or simple nephrectomy since nephron-sparing surgery has been linked to positive outcomes with no compromise in overall survival rates. Finally, in cases where WT has metastasized or is anaplastic, we recommend use of IMAT or VMAT over AP-PA radiotherapy because of their ability to reduce radiation dose to nonneoplastic tissues and organs. Although these treatments are widely practiced to manage WT, there are many potential therapies that can further decrease mortality and morbidity. The prospective use of Wnt/ $\beta$-catenin pathway inhibitors, miRNA, M6620, and stem cell therapy in treating this disease is particularly intriguing. Modern advances in WT treatment may lead to more effective curative measures, ultimately decreasing mortality and relapse rates, and serious therapy-associated side effects. By continuing to address these outcomes, researchers will facilitate the development of superior therapeutics that will ultimately increase both the survival and quality of life in patients affected by WT. 


\section{REFERENCES}

1. Szychot E, Apps J, Pritchard-Jones K. Wilms' tumor: Biology, diagnosis and treatment. Transl Pediatr. 2014;3:12-24. doi:10.3978/j.issn.2224-4336.2014.01.09

2. Lee SH, Paik KH, Sung KW, et al. Renal function after tandem high-dose chemotherapy and autologous stem cell transplantation in children with Wilms tumor. Pediatr Transplant. 2011;15(8):855-860. doi:10.1111/j.1399-3046.2011.01594.x

3. Popov SD, Vujanic GM, Sebire NJ, et al. Bilateral Wilms tumor with TP53-related anaplasia. Pediatr Dev Pathol. 2013;16(3):217-223. doi:10.2350/12-08-1245-cr.1

4. Perotti D, Hohenstein P, Bongarzone I, et al. Is Wilms Tumor a Candidate Neoplasia for Treatment with WNT/ -Catenin Pathway Modulators?-A Report from the Renal Tumors BiologyDriven Drug Development Workshop. Mol Cancer Ther. 2013;12(12):2619-2627. doi:10.1158/ 1535-7163.mct-13-0335

5. Ehrlich A. Wilms Tumor Treatment Protocols. DynaMed. Published online 2018. https://www.dynamed.com/management/wilms-tumor-treatment-protocols

6. Ehrlich P. Combination Chemotherapy and Surgery in Treating Young Patients with Wilms Tumor. Children's Oncology Group. 2019;Clinical Trials.gov Identifier: NCT00945009; 2019.

7. Bhatnagar S, Bhatnagar S. Management of Wilms' tumor: NWTS vs SIOP.J Indian Assoc Pediatr Surg. 2009;14(1):6. doi:10.4103/0971-9261.54811

8. Li MJ, Zhou YB, Huang Y, et al. A Retrospective Study of the Preoperative Treatment of Advanced Wilms Tumor in Children with Chemotherapy versus Transcatheter Arterial Chemoembolization Alone or Combined with Short-term Systemic Chemotherapy. Journal of Vascular and Interventional Radiology. 2011;22(3):279-286. doi:10.1016/j.jvir.2010.11.025

9. Taskinen S, Leskinen O, Lohi J, Koskenvuo M, Taskinen M. Effect of Wilms tumor histology on response to neoadjuvant chemotherapy. Journal of Pediatric Surgery. 2019;54(4):771-774. doi:10.1016/j.jpedsurg.2018.05.010

10. Vujanić GM, D’Hooghe E, Popov SD, Sebire NJ, Kelsey A. The effect of preoperative chemotherapy on histological subtyping and staging of Wilms tumors: The United Kingdom Children's Cancer Study Group (UKCCSG) Wilms tumor trial 3 (UKW3) experience. Pediatr Blood Cancer. 2018;66(3):e27549. doi:10.1002/pbc.27549

11. Jia X, Lai C, Pan HP, Zhou HC, Yang L, Fei ZH. Comparative evaluation of preoperation transcatheter arterial chemoembolization in children with advanced wilms tumor. Zhonghua yi xue za zhi. 2019;99(15):1147-1151. doi:10.3760/cma.j.issn.0376-2491.2019.15.006

12. Vanden Berg RNW, Bierman EN, Noord MV, Rice HE, Routh JC. Nephron-sparing surgery for wilms tumor: A systematic review. Urol Oncol. 2016;34(1):24-32. doi:10.1016/ j.urolonc.2015.07.003

13. Meisel JA, Guthrie KA, Breslow NE, Donaldson SS, Green DM. Significance and management of computed tomography detected pulmonary nodules: A report from the national wilms tumor study group. Int J Radiat Oncol Biol Phys. 1999;44(3):579-585. doi:10.1016/ $\underline{\text { s0360-3016(99)00086-3 }}$

14. Suzuki G, Ogata T, Aibe N, et al. Effective heart-sparing whole lung irradiation using volumetric modulated arc therapy: A case report. J Med Case Reports. 2019;13(1):277. doi:10.1186/s13256-019-2209-2

15. Kalapurakal JA, Gopalakrishnan M, Walterhouse DO, et al. Cardiac-Sparing Whole Lung IMRT in Patients With Pediatric Tumors and Lung Metastasis: Final Report of a Prospective Multicenter Clinical Trial. Int J Radiat Oncol Biol Phys. 2019;103(1):28-37. doi:10.1016/ j.ijrobp.2018.08.034 
16. Hillbrand M, Georg D, Gadner H, Pötter R, Dieckmann K. Abdominal cancer during early childhood: A dosimetric comparison of proton beams to standard and advanced photon radiotherapy. Radiotherapy and Oncology. 2008;89(2):141-149. doi:10.1016/j.radonc.2008.06.012 17. Vogel J, Lin H, Both S, Tochner Z, Balis F, Hill-Kayser C. Pencil beam scanning proton therapy for treatment of the retroperitoneum after nephrectomy for wilms tumor: A dosimetric comparison study. Pediatr Blood Cancer. 2017;64(1):39-45. doi:10.1002/pbc.26176

18. Guerreiro F, Seravalli E, Janssens GO, van den Heuvel-Eibrink MM, Lagendijk JJW, Raaymakers BW. Potential benefit of MRI-guided IMRT for flank irradiation in pediatric patients with wilms' tumor. Acta Oncol. 2019;58(2):243-250. doi:10.1080/0284186x.2018.1537507

19. Koike J, Takagi A, Miwa T, Hirai M, Terada M, Katoh M. Molecular cloning of Frizzled-10, a novel member of the Frizzled gene family. Biochem Biophys Res Commun. 1999;262(1):39-43. doi:10.1006/bbrc.1999.1161

20. Dijksterhuis JP, Petersen J, Schulte G. WNT/Frizzled signalling: Receptor-ligand selectivity with focus on FZD-G protein signalling and its physiological relevance: IUPHAR Review 3. BrJ Pharmacol. 2014;171(5):1195-1209. doi:10.1111/bph.12364

21. Stamos JL, Weis WI. The $\beta$-Catenin Destruction Complex. Cold Spring Harb Perspect Biol. 2013;5(1):a007898-a007898. doi:10.1101/cshperspect.a007898

22. MacDonald BT, Tamai K, He X. Wnt/beta-Catenin Signaling: Components, Mechanisms, and Diseases. Dev Cell. 2009;17(1):9-26. doi:10.1016/j.devcel.2009.06.016

23. Clevers H. Wnt/beta-Catenin Signaling in Development and Disease. Cell. 2006;127(3):469-480. doi:10.1016/j.cell.2006.10.018

24. Vilchez V, Turcios L, Gedaly R. Targeting Wnt/ $\beta$-catenin pathway in hepatocellular carcinoma treatment. World J Gastroenterol. 2016;22(2):823-832. doi:10.3748/wjg.v22.i2.823

25. Rivera MN, Kim WJ, Wells J, et al. An X chromosome gene, WTX, is commonly inactivated in Wilms tumor. Science. 2007;315(5812):642-645. doi:10.1126/science.1137509

26. Zimmerli D, Hausmann G, Cantù C, Basler K. Pharmacological interventions in the Wnt pathway: Inhibition of Wnt secretion versus disrupting the protein-protein interfaces of nuclear factors. BrJ Pharmacol. 2017;174(24):4600-4610. doi:10.1111/bph.13864

27. Pode-Shakked N, Harari-Steinberg O, Haberman-Ziv Y, et al. Resistance or sensitivity of Wilms' tumor to anti-FZD7 antibody highlights the Wnt pathway as a possible therapeutic target.

Oncogene. 2011;30(14):1664-1680. doi:10.1038/onc.2010.549

28. Nomura M, Rainusso N, Lee YC, et al. Tegavivint and the $\beta$-catenin/ALDH Axis in Chemotherapy-Resistant and Metastatic Osteosarcoma. Oxford Univ Press. 2019;111(11):1-43. doi:10.1093/jnci/djz026

29. Ludwig N, Nourkami-Tutdibi N, Backes $C$, et al. Circulating serum miRNAs as potential biomarkers for nephroblastoma. Pediatr Blood Cancer. 2015;62(8):1360-1367. doi:10.1002/ pbc. 25481

30. Walz AL, Ooms A, Gadd S, et al. Recurrent DGCR8, DROSHA, and SIX Homeodomain Mutations in Favorable Histology Wilms Tumors. Cancer Cell. 2015;27(2):286-297. doi:10.1016/ j.ccell.2015.01.003

31. Gong Y, Zou B, Chen J, et al. Potential five-microRNA signature model for the prediction of prognosis in patients with wilms tumor. Med Sci Monit. 2019;25:5435-5444. doi:10.12659/ $\underline{\text { msm. } 916230}$

32. Ardekani AM, Naeini MM. The Role of MicroRNAs in Human Diseases. Avicenna J Med Biotechnol. 2010;2(4):161-179. 
33. Li T, Zhao P, Li Z, Wang CC, Wang YL, Gu Q. MIR-200c-3p suppresses the proliferative, migratory, and invasive capacities of nephroblastoma cells via targeting FRS2. Biopreserv Biobank. 2019;17(5):444-451. doi:10.1089/bio.2019.0009

34. Glade-Bender J. Selinexor in Treating Younger Patients With Recurrent or Refractory Solid Tumors or High-Grade Gliomas. Children's Oncology Group. ClinicalTrials.gov Identifier: NCT02323880; 2020.

35. Kurmasheva RT, Kurmashev D, Reynolds CP, et al. Initial testing (stage 1) of M6620 (formerly VX-970), a novel ATR inhibitor, alone and combined with cisplatin and melphalan, by the Pediatric Preclinical Testing Program. Pediatr Blood Cancer. 2017;65(2):e26825. doi:10.1002/ pbc. 26825

36. Thomas A, Redon CE, Sciuto L, et al. Phase I study of ATR inhibitor M6620 in combination with topotecan in patients with advanced solid tumors. J Clin Oncol. 2018;36(16):1594-1602. doi:10.1200/jco.2017.76.6915

37. Malogolowkin MH, Hemmer MT, Le-rademacher J, et al. Guilcher G, et al retrospective analysis. 2018;52:1549-1555. doi:10.1038/bmt.2017.178. Outcomes 